the alkaline type of generator as the source of nascent hydrogen. Aluminum and dil. potassium hydroxide solution serve best for the formation of large quantities of germanium hydride, and sodium amalgam is best for small quantities.

3. The apparatus described, which is a modification of the ordinary Marsh apparatus, permits the formation of a richer mixture of the hydride than has hitherto been prepared and still further increases the delicacy of the hydride test.

4. The decomposition temperature of the hydride lies between $340^{\circ}$ and $360^{\circ}$; deposition of the mirror is best obtained below a red heat.

The reverse reaction involving the loss of germanium in hydrogen has been quantitatively estimated and shows the magnitude of the loss suffered by this metal in hydrogen at $750-800^{\circ}$.

5 . The dissimilarity of films of germanium and arsenic as obtained in the Marsh test, is shown by spectroscopic examination of the light which these films transmit and the thickness of various mirrors has been determined.

6. Study of the composition of the compound described by Voegelen as silver germanide plainly shows that this precipitate is variable in composition and may consist of nearly all free silver with very little combined germanium, or nearly pure silver germanide, depending upon the method used in the preparation of the hydride.

7. The conditions are stated which favor the formation of tetrasilvergermanide, $\mathrm{Ag}_{4} \mathrm{Ge}$, and also those which result in the formation of free silver and soluble germanic acid by the action of the hydride upon a silver salt.

Phit,adel,phia, Pennsyl,vania

|Contribution from the School of Chemical Enginearing Practice, MassachusetTS Institute of Technology, No. 20]

\title{
THE SOLUBILITY OF SULFUR DIOXIDE IN SUSPENSIONS OF CALCIUM AND MAGNESIUM HYDROXIDES
}

By Wm. Thompson Smith and Reginald B. Parkhurst

Received June 1, 1922

From the time of the investigation and original patents of B. C. Tilghman, ${ }^{1}$ the development and use of the sulfite process in pulp making has steadily progressed until to-day it is the most important method of making chemical wood pulp. Practice has shown that wide variations in the quality of the product are to be expected as the quality of the cooking liquor varies, yet any attempts at the control of the latter, either by the design of apparatus or in the process of manufacture, have necessarily

1 Titghman, Brit. pat. 2,926 (1866); Ger. pat., March 31, 1867; U. S. pat., October $26,1867$. 
been largely empirical due to the lack of data concerning the solubility of sulfur dioxide in the calcium and magnesium hydroxide suspensions used. Practically all calculations have been based upon the data presented by $\operatorname{Sims}^{2}$ and others on the solubility of sulfur dioxide in water; these data have to a certain extent been verified in the investigation here reported.

The following factors, as listed by Hopkinson, ${ }^{3}$ affect and control the solubility of sulfur dioxide in suspensions of lime and magnesia: (1) alkalinity of lime suspension; (2) strength of gas (partial pressure over the solution under equilibrium conditions); (3) temperature of solution; (4) proportions of lime and magnesia.

In practice the suspension is made up from a dolomitic lime ranging from 30 to $40 \%$ of magnesium oxide and of a strength such that the total alkalinity as determined by direct titration commonly approximates 0.5 equivalent per liter. The gas used, produced as needed, is from sulfur burners and therefore, under good operating conditions when mixed with gas returned from the digesters, has an average strength in sulfur dioxide of 25 to $30 \%$, allowing a partial pressure over the finished bisulfite liquor of not over 250 millimeters of mercury. The temperature during the manufacture of the liquor is normally kept as low as possible but is generally not closely controlled and is subject to considerable variation. These facts taken from normal practice have fixed the limits for the ranges in which the effects of the variables listed above have been investigated. Solutions in all proportions of calcium and magnesium varying in total alkalinity from zero to one equivalent per liter and at temperatures between $5^{\circ}$ and $60^{\circ}$ have been used with the equilibrium partial pressure of sulfur dioxide over the solution as high as $760 \mathrm{~mm}$.

A careful consideration, from the standpoint of accuracy and of simplicity of apparatus and operation, of methods for the determination of gas solubility to be found completely described in the literature as well as in the unpublished work of Pitman, ${ }^{4}$ King, ${ }^{5}$ and Hopkinson ${ }^{3}$ led to the adoption of the method and apparatus to be described as follows. Two portions of solvent were placed in separate parts of the same closed system and allowed to attain the desired temperature. After evacuating the system as completely as possible and sweeping out any remaining gases by allowing the solutions to boil for a few seconds, the absorption apparatus was closed and an initial pressure reading made. After closing off one portion of the solvent, sulfur dioxide was admitted to the system and the second portion of the solvent was thoroughly and continually agitated

${ }^{2}$ Sims, Ann., 118, 333 (1861); Am. Chem. J., 14, 1 (1862).

${ }^{3}$ Hopkinson, Mass. Inst. Techn. Chem. Eng. Thesis, 1921.

4 Pitman, ibıd., 1916.

${ }^{5} \mathrm{King}$, ibid. (Industrial), 1916. 
by shaking until no further rapid change in the manometer was noticeable. To guard against the effect of any possible inert gas having been admitted as an impurity in the sulfur dioxide used, the system was again quickly evacuated and any such gases driven out by the evolution of sulfur dioxide from the solution. Now, the absorption system being isolated, both portions of the solvent were opened to the action of the gas. A drop in pressure was of course immediately noticeable and there were then in the system two portions of solvent, one oversaturated and the other undersaturated with respect to sulfur dioxide at the pressure then exerted. Continuous and thorough agitation by shaking was then maintained for a period varying in different runs from 20 minutes to $1 \frac{1}{2}$ hours, following which a final pressure reading was made and the contents, gaseous and liquid, of the flasks analyzed by titration with standard iodine and sodium thiosulfate solutions.

Such a method has a number of advantages worthy of special enumeration as follows: (1) no analysis of the gas used is necessary over each flask, as would have been required in the case of bubbling mixtures of sulfur dioxide and some inert gas through the solvent, by this means controlling and measuring the partial pressure of the sulfur dioxide; (2) the effect of barometric variation is eliminated, by the use of pressure differences only in the calculations; (3) no measurement of the volume of gas used is necessary nor does the change in the volume of the system through the change in the manometer level have any effect; (4) no accurate control or measurement of any rate of flow is necessary; (5) the equilibrium concentration is approached from both sides allowing the use of an arithmetric mean to obtain accurate data; (6) no controlled variation in temperature is required in the procedure; (7) sulfur dioxide pressures in excess of one atmosphere may readily be obtained and handled; (8) since the sulfur dioxide is determined in both gaseous and liquid contents of the flasks, there is no chance of loss of sulfur dioxide or of inaccurate measurement of its solubility on this account.

The apparatus used was constructed essentially as follows: sulfur dioxide, from a tank of the liquefied gas ${ }^{6}$ was admitted through a connection of rubber pressure tubing to one arm of the inlet of the absorption system. To the other arm was attached a Richards pump. At the inlet was a glass stopcock which served, during the progress of a run, to isolate the system effectively. Immediately below the stopcock a side arm provided means of connecting the absorption flasks to the rest of the system. Below this side arm was located a mercury-filled $\mathrm{U}$-tube serving as a manometer in the arms of which the height of the mercury might be read by the use of a sliding rider upon a meter stick

- Provided for the purpose of this investigation by the Virginia Smelting Company of Norfolk, Virginia. 
permanently attached to the manometer support. By means of pressure tubing, a multiple header, entirely of glass and provided with 12 outlets, was attached to the above-mentioned side arm. Twelve flasks containing the solvent were placed in compartments in a weighted basket of wire screen provided with handles for convenience in shaking and with a cover for holding the flasks securely in place. These flasks were connected by short lengths of rubber pressure tubing, each to one outlet of the multiple header. Finally, the basket and its contents were immersed in a water-bath serving as a thermostat of sufficient size to allow the basket to be shaken without removal. Sufficient freedom of motion for this agitation was allowed by the rubber tubing connection.

For the actual determination of the quantity of sulfur dioxide dissolved, screw clamps were closed on the rubber connections between each flask and the header, after which the header was removed. A separatory funnel containing an excess of iodine solution was then connected to the flask, the contents of which were to be analyzed, and the screw clamp opened. The iodine solution was allowed to run in and the funnel washed with distilled water, in such a manner as to maintain, until the funnel was clean, a liquid seal over the flask. The flask was then opened and the excess of iodine was titrated in the usual manner with sodium thiosulfate solution. The solutions used were prepared as usual, the iodine being standardized against the thiosulfate which in turn was standardized against resublimed iodine and against a solution the strength of which had been determined by the use of pure copper as a standard. The results obtained were in satisfactory agreement varying by less than $0.5 \%$.

Various writers report difficulties with this determination unless the sulfur dioxide solution be added to the excess of iodine solution. Such difficulties may arise from either or both of the following two sources. In the first place, when iodine is added, sulfur dioxide may be lost by ebullition. This difficulty is entirely eliminated by our method. Second, hydriodic acid as formed may enter into a secondary reaction with free sulfurous acid, generally explained as by Volhard. ${ }^{7}$

$$
\begin{aligned}
& 2 \mathrm{SO}_{2}+21_{z}+4 \mathrm{H}_{2} \mathrm{O}=4 \mathrm{HI}+2 \mathrm{H}_{2} \mathrm{SO}_{4} \\
& \mathrm{SO}_{2}+2 \mathrm{HI}=2 \mathrm{H}_{2} \mathrm{O}+\mathrm{S}+21_{2}
\end{aligned}
$$

This would mean that less than the expected amount of iodine would be required for a given quantity of sulfur dioxide. The secondary reaction may be avoided, naturally, if the formation of hydriodic acid be not allowed. The use of sodium hydrogen carbonate is recommended by Rupp ${ }^{8}$ to form sodium iodide and free carbon dioxide. For a trial of this remedy, a saturated solution of sodium hydrogen carbonate was prepared and used in test determinations, a volume of this solution approximately equal

${ }^{7}$ Volhard, Ann., 242, 94 (1887).

' Rupp, Ann. Physik. Bl., 35, 3694 (1902). 
to that of the iodine solution being first added from the separatory funnel. The fact that results obtained with and without the use of the bicarbonate are in agreement seems to point to all such analytical errors being due, in previous work, to the first source mentioned above and eliminated in this investigation.

Throughout the results as reported the solubility of sulfur dioxide as free sulfurous acid is expressed not in grams per unit quantity of solution but rather as per unit quantity of water added. Therefore these results are, strictly speaking, not immediately applicable where data are at hand

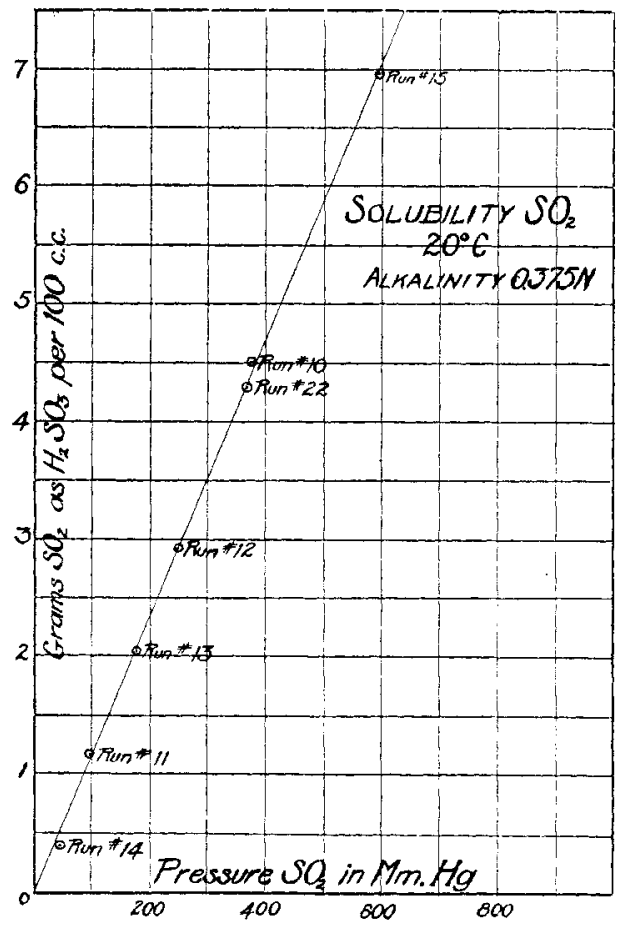

Fig. 1. merely for the volume of solt1tion. On the other hand, the volume change due to the solution of calcium or magnesium hydrogen sulfite in water in the concentrations worked with are very slight. For all practical purposes the correction is negligible.

In the results of this investigation a volume change in the solution would theoretically affect the solubility as reported for the reason that the gas space would be decreased by exactly the increase in liquid volume. As a correction for water vapor, this gas space was assumed to be filled with pure sulfur dioxide at a pressure determined by the difference between final and initial pressure readings and, the volume being known from the flask calibration, the iodine equivalent of the gas was calculated assuming the strict applicability of the gas laws. This equivalent, influenced both by any volume change and by possible faulty flask calibration, subtracted from the total titration value gave the total amount of sulfur dioxide in the solution. A further subtraction of the amount of sulfur dioxide equivalent to the base present gave the quantity of free sulfurous acid.

From a consideration of the volume change to be expected by the solution of a salt in water in the proportion of one equivalent per liter (the highest concentration used), it is at once obvious that in approximately $20 \mathrm{cc}$. of water as used the actual magnitude of the volume change would be far less than any error probable in the calibration of the flasks the volume 
of each of which was approximately $250 \mathrm{cc}$. To show in a striking manner the very small effect of either of these errors, let us exaggerate the probable calibration error and pick those conditions under which its effect would be most serious. Let us assume that the calibration of Flasks 11 and 12 gave values in each case $5 \mathrm{cc}$. less than the actual capacity. This would amount to a total error of approximately 10 parts in 500 or $2 \%$ of the iodine equivalent of the sulfur dioxide present in the flask and undissolved. In Run 18 at $60^{\circ}$ the average gas correction amounted to $4.28 \mathrm{cc}$. of the iodine solution. In this case the average iodine equivalent of the solution alone amounted to $41.78 \mathrm{cc}$. The error introduced by the assumed incorrect calibration would amount to $0.08 \mathrm{cc}$. The iodine equivalent of the alkali present was $39.67 \mathrm{cc}$., thus leaving the iodine equivalent of the free sulfur dioxide in the solution as $2.11 \mathrm{cc}$. The final error would therefore be less than $4 \%$.

Let it now be considered that the maximum probable calibration error is equal to or less than $1 \mathrm{cc}$. It is seen that the maximum percentage error to be expected amounts to less than $1 \%$ in the worst case, namely, at the highest temperature and total alkalinity used, and consequently the lowest concentration of sulfur dioxide as free sulfurous acid. It was, there-

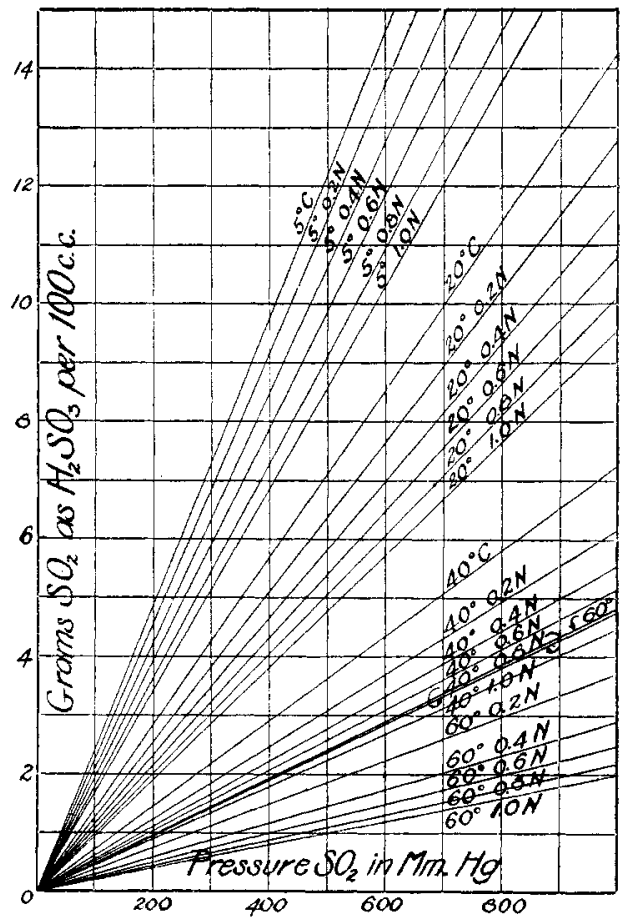

Fig. 2. fore, considered justifiable to neglect the effect of these errors in the calculation of all results.

The meter stick used was found to be sufficiently accurate to cause a maximum error in pressure readings of $0.5 \mathrm{~mm}$. This error is, therefore, in all cases small enough to have no appreciable effect upon the results as reported. Other errors in the pressure determinations will theoretically arise from the neglect in the later runs of the variations in the vapor pressure of water over solutions whose normality in alkali ranged from zero to one. Such variation is extremely small especially when compared with the total pressure used and its neglect is clearly justifiable.

In Run 16, determining the effect of varying proportions of calcium 
and magnesium in solutions of the same total alkalinity, 5 values showing a maximum variation from the mean of $1 \%$ satisfied the investigators as to the effect of this variable. No check determinations were made at other temperatures or total alkalinities since the values used in this case were chosen at random and a consideration of the law of probabilities justifies the general application of the results so obtained.

Among the objections to the procedure as developed in this investigation may be included the error due to evaporation losses incurred when the solutions were boiled to displace inert gases in the system. Such losses were absolutely negligible as is shown by comparison with Sims' data of the values obtained at different temperatures and pressures for the solubility of

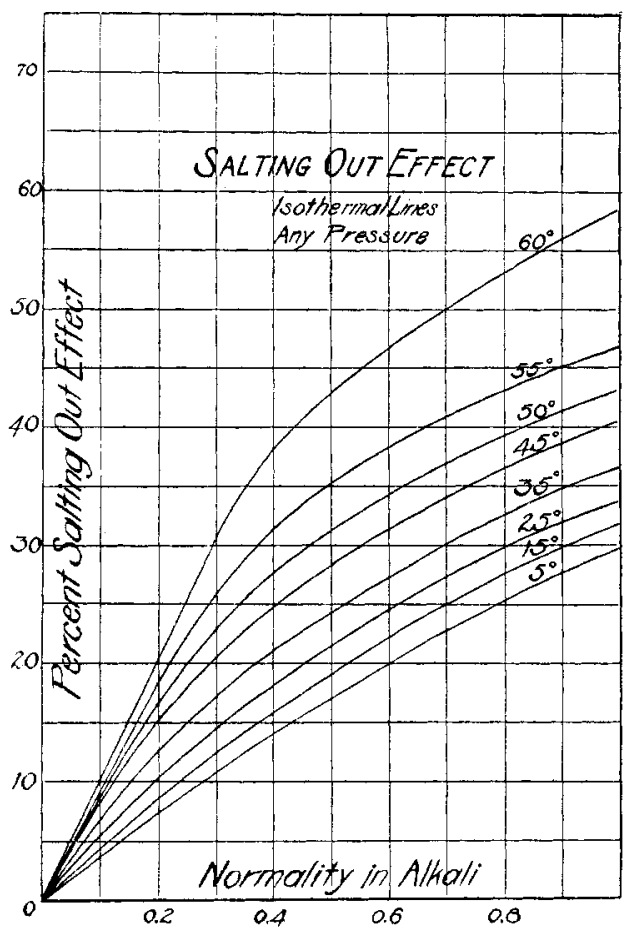

Fig. 3. sulfur dioxide in water. 'The present results are found to be consistently slightly below those of Sims and this fact is readily and completely explainable by the statement that Sims' data were obtained, in the pressure ranges here used, by allowing in all cases a supersaturated solution to come to substantial equilibrium in his apparatus. Therefore, the very slight variation between Sims' data and the results herein presented entirely precludes the possibility of any significant errors having arisen from evaporation losses.

When two compounds, ionizing to give a common ion, are present in the same solution, it is a well-known fact that each acts to repress the ionization of the other. When, furthermore, the solution is saturated with respect to one of the compounds (as in this case with respect to sulfurous acid), the solubility product or product of the ionic concentrations of any compound under given conditions being constant, the presence of a common ion from the second compound acts to reduce the solubility of the first producing a salting-out effect. We may now define, for this special case and as the units in which part of the experimental results are expressed, the percentage salting-out effect to be 100 times the ratio of the difference between the solubility of sulfur dioxide in water and the solubility of sulfur dioxide 
as free sulfurous acid in the solution in question to the solubility of sulfur dioxide in water under the same conditions of temperature and pressure.

In conclusion, the results of the above outlined experimental investigation may be stated as follows.

1. The concentration of free sulfur dioxide or sulfurous acid in water and in calcium and magnesium hydrogen sulfite solutions is exactly and directly proportional to the partial pressure of sulfur dioxide in the gases above the solution at all temperatures, alkali concentrations and pressures within the range investigated.

2. At any definite alkalinity and at all pressures within the range investigated, the ratio of the difference between the solubility of sulfur dioxide in water and in the solution and the solubility of the gas in water (the salting-out effect) increases with the temperature and at a rate more than in proportion to the temperature increase.

3. At any definite temperature and at all pressures within the range investigated, the salting-out effect (as defined above) increases with the total alkalinity and at a rate less than in proportion to the increase in alkali concentration.

4. Varying proportions of calcium and magnesium have no effect upon the concentration of free sulfur dioxide or sulfurous acid at any constant molal concentration.

\begin{tabular}{|c|c|c|c|c|c|c|}
\hline Run & $\begin{array}{l}\text { Press. of } \\
\mathrm{SO}_{2} \text { in } \\
\mathrm{mm} \text { of } \mathrm{Hg} .\end{array}$ & $\begin{array}{c}T \\
{ }^{\circ} \mathrm{C} .\end{array}$ & Flasks & $\begin{array}{c}\text { Norm. of sol. } \\
\text { in alkali } \\
\text { at start } \\
N\end{array}$ & $\begin{array}{l}\text { dissolved } \\
\text { G. per } 100 \mathrm{cc} \text {. } \\
\text { of water used }\end{array}$ & $\begin{array}{l}\text { G. per } \\
100 \mathrm{cc} \text {. of } \\
\text { water used }\end{array}$ \\
\hline 8 & 224.4 & 20 & A,B & 0 & 3.34 & 3.34 \\
\hline 9 & 198.6 & 20 & $A, B$ & 0 & 2.97 & 2.97 \\
\hline 10 & 374.1 & 20 & $A, B$ & $0.375(\mathrm{Ca})$ & 6.91 & 4.51 \\
\hline 11 & 97.1 & 20 & $A, B$ & $0.375(\mathrm{Ca})$ & 3.56 & 1.16 \\
\hline 12 & 250.3 & 20 & $A, B$ & $0.375(\mathrm{Ca})$ & 5.32 & 2.92 \\
\hline 13 & 177.9 & 20 & $\mathrm{~A}, \mathrm{~B}$ & $0.375(\mathrm{Ca})$ & 4.45 & 2.05 \\
\hline 14 & 46.6 & 20 & $\mathrm{~A}, \mathrm{C}$ & $0.375(\mathrm{Ca})$ & 2.80 & 0.40 \\
\hline 15 & 595.3 & 20 & $\mathrm{~A}, \mathrm{C}$ & $0.375(\mathrm{Ca})$ & 9.36 & 6.96 \\
\hline \multirow[t]{6}{*}{16} & 239.0 & 20 & 1,2 & $0.5(\mathrm{Ca})$ & 5.72 & 2.52 \\
\hline & & & 3,4 & $\begin{array}{l}0.4(\mathrm{Ca}) \\
0.1(\mathrm{Mg})\end{array}$ & 5.75 & 2.55 \\
\hline & & & 5,6 & $\begin{array}{l}0.3(\mathrm{Ca}) \\
0.2(\mathrm{Mg})\end{array}$ & 5.61 & 2.41 \\
\hline & & & 7,8 & $\begin{array}{l}0.2(\mathrm{Ca}) \\
0.3(\mathrm{Mg})\end{array}$ & 5.70 & 2.50 \\
\hline & & & 9,10 & $\begin{array}{l}0.1(\mathrm{Ca}) \\
0.4(\mathrm{Mg})\end{array}$ & 5.76 & 2.56 \\
\hline & & & 11,12 & $0.5(\mathrm{Mg})$ & 5.75 & 2.55 \\
\hline \multirow[t]{2}{*}{17} & 474.8 & 5 & 1,2 & 0 & 11.55 & 11.55 \\
\hline & & & 3,4 & $0.2(\mathrm{Mg})$ & 12.11 & 10.83 \\
\hline
\end{tabular}




\begin{tabular}{|c|c|c|c|c|c|c|}
\hline \multicolumn{7}{|c|}{ TABULAR Results (Conitinued) } \\
\hline \multirow[t]{6}{*}{ Run } & $\begin{array}{l}\text { Press. of } \\
\mathrm{SO}_{2} \text { in } \\
\mathrm{mm} \text {. of } \mathrm{Hg}\end{array}$ & $T$ & Flasks & $\begin{array}{l}\text { Norm. of sol. } \\
\text { in alkali } \\
\text { at start }\end{array}$ & $\begin{array}{c}\text { Total } \mathrm{SO}_{2} \\
\text { dissolved } \\
\text { G. per } 100 \mathrm{cc} \text {. } \\
\text { of water used }\end{array}$ & $\begin{array}{l}\text { Free } \mathrm{SO}_{2} \\
\text { G. per } \\
100 \mathrm{cc} \text {. of } \\
\text { water used }\end{array}$ \\
\hline & & ${ }^{\circ} \mathrm{C}$ & & $N$ & & \\
\hline & & & 5,6 & $0.4(\mathrm{Mg})$ & 12.61 & 10.05 \\
\hline & & & 7,8 & $0.6(\mathrm{Mg})$ & 13.21 & 9.37 \\
\hline & & & 9,10 & $0.8(\mathrm{Mg})$ & 13.68 & 8.56 \\
\hline & & & 11,12 & $1.0(\mathrm{Mg})$ & 14.65 & 8.25 \\
\hline \multirow{6}{*}{18} & 175.7 & 60 & 1,2 & 0 & 0.84 & 0.84 \\
\hline & & & 3,4 & $0.2(\mathrm{Mg})$ & 1.89 & 0.61 \\
\hline & & & 5,6 & $0.4(\mathrm{Mg})$ & 3.11 & 0.55 \\
\hline & & & 7,8 & $0.6(\mathrm{Mg})$ & 4.24 & 0.40 \\
\hline & & & 9,10 & $0.8(\mathrm{Mg})$ & 5.52 & 0.40 \\
\hline & & & 11,12 & $1.0(\mathrm{Mg})$ & 6.74 & 0.34 \\
\hline \multirow[t]{6}{*}{19} & 306.4 & 40 & 1,2 & 0 & 2.21 & 2.21 \\
\hline & & & 3,4 & $0.2(\mathrm{Mg})$ & 3.16 & 1.88 \\
\hline & & & 5,6 & $0.4(\mathrm{Mg})$ & 4.30 & 1.74 \\
\hline & & & 7,8 & $0.6(\mathrm{Mg})$ & 5.40 & 1.56 \\
\hline & & & 0,10 & $0.8(\mathrm{Mg})$ & 6.65 & 1.53 \\
\hline & & & 11,12 & $1.0(\mathrm{Mg})$ & 7.80 & 1.40 \\
\hline 20 & 1107.8 & 20 & 1,2 & 0 & 15.01 & 15.01 \\
\hline \multirow[t]{6}{*}{21} & 373.8 & 40 & 1,2 & $1.0(\mathrm{Mg})$ & 7.95 & 1.55 \\
\hline & & & 3,4 & $0.8(\mathrm{Mg})$ & 6.81 & 1.69 \\
\hline & & & 5,6 & $0.6(\mathrm{Mg})$ & 5.89 & 2.05 \\
\hline & & & 7,8 & $0.4(\mathrm{Mg})$ & 4.45 & 1.89 \\
\hline & & & 9,10 & $0.2(\mathrm{Mg})$ & 3.54 & 2.26 \\
\hline & & & 11,12 & 0 & 2.68 & 2.68 \\
\hline \multirow[t]{6}{*}{22} & 360.6 & 20 & 1,2 & 0 & 5.22 & 5.22 \\
\hline & & & 3,4 & $0.2(\mathrm{Mg})$ & 5.95 & 4.67 \\
\hline & & & 5,6 & $0.4(\mathrm{Mg})$ & 6.70 & 4.30 \\
\hline & & & 7,8 & $0.6(\mathrm{Mg})$ & 7.98 & 4.14 \\
\hline & & & 9,10 & $0.8(\mathrm{Mg})$ & 8.82 & 3.68 \\
\hline & & & 11,12 & $1.0(\mathrm{Mg})$ & 9.90 & 3.48 \\
\hline
\end{tabular}

This article reports an investigation of the solubility of sulfur dioxide in water, milk of lime, and milk of magnesia and includes a description of the apparatus used. Partial pressures of sulfur dioxide up to $760 \mathrm{~mm}$., temperatures from $5^{\circ}$ to $60^{\circ}$, all proportions of calcium and magnesium, and suspensions of total alkalinity up to one equivalent per liter were used in the experimental work.

1. The concentration of sulfur dioxide as sulfurous acid is found to be proportional to its partial pressure.

2. With solutions of the same alkali concentration, the percentage salting-out effect increases with, but more than in proportion to, the temperature.

3. With solutions of constant temperature, the percentage salting-out 
effect increases with, but less than in proportion to, the alkali concentration.

4. Varying proportions of calcium and magnesium have no effect upon the equilibrium concentration of sulfur dioxide as sulfurous acid.

Cambridge 39, MassachusetTs

[CONTribution from the Chemical Laboratory of the University of Illinois] OBSERVATIONS ON THE RARE EARTHS. XII. THE ATOMIC WEIGHT OF LANTHANUM

By B. S. Hopkins and F. H. DRiggs

Received June 16,1922

During the progress of the work upon the rare-earth group, there was received at this laboratory a shipment of $182 \mathrm{~kg}$. of sodium rare earth sulfates, which was generously supplied by the Welsbach Company of Gloucester, New Jersey, and its chief chemist, Dr. H. S. Miner. The early treatment of this material' consisted in the removal of cerium by the method proposed by James and Pratt, ${ }^{2}$ and fractional crystallization as the double magnesium nitrate. This work was done by Dr. Edward Wichers. Later the fractions from the insoluble end of the series which contained lanthanum and praseodymium were subjected to a long series of fractional crystallizations as the ammonium-rare earth nitrates under the care of Dr. E. W. Engle. The fractionation was continued until the first 20 fractions failed to show the slightest trace of praseodymium in their absorption spectra, when viewed through a $10 \mathrm{~cm}$. Iayer of concentrated solution.

\section{Purification of Material}

Portions of Fractions 7 and 8 of the double ammonium nitrate series were diluted and treated with a hot dil. solution of oxalic acid. The precipitate was washed, dried and ignited to the oxide in an electric muffle. The oxide was redissolved in nitric acid, diluted to $2000 \mathrm{cc}$. and lanthanum hydroxide precipitated by distilling ammonia vapors into the solution. This precipitate was washed thrice by decantation and redissolved in nitric acid. The alternate precipitation of hydroxide and oxalate was repeated four times, the last two precipitations being carried out in conductivity water. The ignited oxide was then suspended in conductivity water and dissolved by passing over it the vapor obtained by distillation of C. P. hydrochloric acid from a quartz flask.

A portion of Fraction 8 of this series was sent to the Bureau of Standards where it was examined spectroscopically by the use of 4 gratings, 1 of 7,500 lines per inch for the infra-red region, 1 of 10,000 lines per inch and

1 See Hopkins and Kremers, Bur. Standards Sci. Papers, 421, Part II (1921).

2 James and Pratt, This Journal, 33, 1326 (1911). 